\title{
Brain Plasticity and Stroke Recovery
}

\author{
Human Motor Control Section, NINDS, NIH Mark Hallett
}

Stroke may lead to significant disability. Many patients do improve spontaneously, at least to some extent, and after the acute period the principal mechanism appears to be brain plasticity. The task for rehabilitation is to improve on spontaneous recovery, and this might well be accomplished by manipulating plasticity. Plasticity is the capability of the brain to undergo change. This has been demonstrated in humans most clearly with deafferentation, motor learning, and in response to brain injury. Spontaneous recovery after stroke leads to plastic changes in both the damaged hemisphere and the undamaged hemisphere (ipsilateral to the weakened body part). Generally, better improvement will result with plasticity of the damaged hemisphere. The undamaged hemisphere seems to play a role early after stroke and achieves an important role if the damaged hemisphere does not recovery well. One important principle for rehabilitation is that use of a body part enhances its representation in brain. Thus, physical therapy should help if there is attention to the weakened extremity, and this has been demonstrated by the multiple successes of constraint-induced movement therapy. Physiological studies do indeed show enhancement of function in the damaged hemisphere after such interventions. Other techniques likely use the same principle such as neuromuscular electrical stimulation, robot-enhanced training and virtual reality training. Bilateral, symmetical arm movement training also helps, and this may work by enhancement of function of the undamaged hemisphere. Sensory stimulation enhances plasticity and can be delivered in a number of ways, from passive movement to cutaneous stimulation with transcutaneous electrical nerve stimulation and even acupuncture. Neuromuscular electrical stimulation may also work in part by enhancing sensory feedback. Reduction of inhibition enhances plasticity. Originally demonstrated at the cellular level, this has been shown to be relevant to human biology with the deafferentation model. Our group has now demonstrated that this can be efficacious in stroke patients. We studied patients with weak hands and more preserved proximal arm function, with a goal of taking over some of the proximal representation in the brain for use by the hand. We coupled anesthesia of the proximal arm with exercise of the hand and showed that this increases hand function more than exercise alone. Pharmacological agents can enhance plasticity by several mechanisms. There has been considerable work with amphetamine. Other agents, such as fluoxetine, methylphenidate and levodopa, have also been shown to be of use. There is considerably more work to do in this area, and the future is promising. 